\title{
Turnpike Properties for Dynamical Systems Determined by Differential Inclusions
}

\author{
Alexander J. Zaslavski \\ Department of Mathematics, Technion-Israel Institute of Technology, Haifa 32000, Israel; ajzas1@technion.ac.il

\begin{abstract}
In this paper, we study the turnpike phenomenon for trajectories of continuous-time dynamical systems generated by differential inclusions, which have a prototype in mathematical economics. In particular, we show that, if the differential inclusion has a certain symmetric property, the turnpike possesses the corresponding symmetric property. If we know a finite number of approximate trajectories of our system, then we know the turnpike and this information can be useful if we need to find new trajectories of our system or their approximations.
\end{abstract}

Keywords: differential inclusions; global attractor; set-valued mapping; turnpike

MSC: 34D30; 34D45; 37B25; 49J53; 93D20

Citation: Zaslavski, A.J. Turnpike Properties for Dynamical Systems Determined by Differential Inclusions. Symmetry 2021, 13, 2326. https:// doi.org/10.3390/sym13122326

Academic Editor: José Carlos R. Alcantud

Received: 15 November 2021 Accepted: 29 November 2021 Published: 4 December 2021

Publisher's Note: MDPI stays neutral with regard to jurisdictional claims in published maps and institutional affiliations.

Copyright: (c) 2021 by the authors. Licensee MDPI, Basel, Switzerland. This article is an open access article distributed under the terms and conditions of the Creative Commons Attribution (CC BY) license (https:// creativecommons.org/licenses/by/ $4.0 /)$.

\section{Introduction}

In [1,2], A. M. Rubinov introduced a discrete disperse dynamical system which is generated by a set-valued self-mapping of a compact metric space. This dynamical system was investigated in [1-7]. It has a prototype in the economic growth theory $[1,2,8,9]$. In particular, it is an abstract extension of the classical von Neumann-Gale model $[1,2,8,9]$. This dynamical system is described by a compact metric space of states and a transition operator which is set-valued. Such dynamical systems correspond to certain models of economic dynamics [1,2,8,9]. More precisely, in [1-3], the description of global attractors for certain dynamical systems was obtained; the uniform convergence of trajectories to global attractors was studied in [4] and the behavior of trajectories under the presence of computational errors was analyzed in [5], while, in [6], analogous results were obtained for systems with a Lyapunov function. These results are collected in our recent book [7].

In the present paper, we study the convergence and structure of trajectories of the continuous-time analog of this dynamical system generated by a differential inclusion. In particular, we show that, if the differential inclusion has a certain symmetric property, its turnpike possesses the corresponding symmetric property.

We introduce a global attractor (turnpike) for our dynamical system which is the closure of the set of all limit points of all trajectories; we show that all trajectories on an infinite interval converge to this set and that all trajectories on finite and sufficiently large intervals spend most of the time in a small neighborhood of the turnpike. If we know a finite number of approximate trajectories of our system, then we know the turnpike and this information can be useful if we need to find new trajectories of our system or their approximations. We believe that our results can be extended to the case of perturbed trajectories of our system.

It should be mentioned that the turnpike phenomenon holds for many problems in various areas of research [7,9-16].

Let $R^{n}$ be the $n$-dimensional Euclidean space equipped with the inner product

$$
\langle x, y\rangle=\sum_{i=1}^{n} x_{i} y_{i}, x=\left(x_{1}, \ldots, x_{n}\right), y=\left(y_{1}, \ldots, y_{n}\right)
$$


which induces the Euclidean norm $\|\cdot\|$ and let $X$ be a nonempty closed set in $R^{n}$ equipped with the relative topology.

Let us denote, by $\mathcal{N}$, the set of all natural numbers. For every point $x \in R^{n}$ and every positive real number $r$, let us put

$$
B(x, r)=\left\{y \in R^{n}:\|x-y\| \leq r\right\}, B^{0}(x, r)=\left\{y \in R^{n}:\|x-y\|<r\right\} .
$$

Let us suppose that $F: X \rightarrow 2^{R^{n}} \backslash\{\varnothing\}$. We define

$$
\operatorname{graph}(F)=\left\{(x, y) \in X \times R^{n}: y \in F(x)\right\} .
$$

The mapping $F$ is upper semicontinuous at a point $x_{0} \in X$ if, for every open set $N$ which contains $F\left(x_{0}\right)$, there is an open neighborhood $\mathcal{M}$ of the point $x_{0}$ in the space $X$ for which $F(\mathcal{M}) \subset N$. $x_{0} \in X$.

The mapping $F$ is upper semicontinuous if it is upper semicontinuous at each point

For the proof of the following result see Proposition 2 of [17].

Proposition 1. Let us assume that $F$ is upper semicontinuous and that $F(z)$ is closed for every $z \in X$. Then, $\operatorname{graph}(F)$ is closed in $X \times R^{n}$.

It is easy to see that the next result is true.

Proposition 2. Let us assume that the set graph $(F)$ is closed in $X \times R^{n}, x_{0} \in X, V$ is a neighborhood of the point $x_{0}$ in the space $X$ and that the set $F(V)$ is bounded. Then, the mapping $F$ is upper semicontinuous at the point $x_{0}$.

Let $-\infty<T_{1}<T_{2}<\infty$. A function $x:\left[T_{1}, T_{2}\right] \rightarrow X$ is called a trajectory if it is absolutely continuous (a. c.) and

$$
x^{\prime}(t) \in F(x(t)), t \in\left[T_{1}, T_{2}\right] \text { almost everywhere (a. e.). }
$$

We denote, by $\mathcal{A}\left(T_{1}, T_{2}\right)$, the collection of all trajectories $x:\left[T_{1}, T_{2}\right] \rightarrow X$.

Let $T_{1} \in R^{1}$. A function $x:\left[T_{1}, \infty\right) \rightarrow X$ is a trajectory if, for every $T_{2}>T_{1}$, its restriction to the interval $\left[T_{1}, T_{2}\right]$ belongs to $\mathcal{A}\left(T_{1}, T_{2}\right)$. we denote, by $\mathcal{A}\left(T_{1}, \infty\right)$, the set of all trajectories $x:\left[T_{1}, \infty\right) \rightarrow X$.

A function $x: R^{1} \rightarrow X$ is a trajectory if, for each pair of numbers $T_{2}>T_{1}$, its restriction to the interval $\left[T_{1}, T_{2}\right]$ belongs to $\mathcal{A}\left(T_{1}, T_{2}\right)$. We denote, by $\mathcal{A}(-\infty, \infty)$, the collection of all trajectories $x: R^{1} \rightarrow X$.

In the sequel, we suppose that the following assumption holds.

(A1) The mapping $F$ is upper semicontinuous and $F(x)$ is a compact, convex set for every point $x \in X$.

In view of Proposition 1 , the set graph $(F)$ is closed in $X \times R^{n}$.

Proposition 3. The mapping $F$ is bounded on bounded sets. In other words, for every positive number $M_{0}$, there is a positive number $M_{1}$ such that

$$
F(x) \subset B\left(0, M_{1}\right) \text { for every point } x \in B\left(0, M_{0}\right) \cap X .
$$

Proof. Let $M_{0}>0$. We show that there exists $M_{1}>0$ such that (2) holds. Let us assume the contrary. Then, for every $k \in \mathcal{N}$, there is

$$
x_{k} \in B\left(0, M_{0}\right) \cap X, y_{k} \in F\left(x_{k}\right)
$$

such that

$$
\left\|y_{k}\right\|>k
$$


In view of (3), we may assume, without loss of generality, that there is a limit

$$
x=\lim _{k \rightarrow \infty} x_{k} .
$$

Since the mapping $F$ is upper semicontinuous, there is an open neighborhood $U$ of the point $x$ in the space $X$ such that, for each point $x \in U$, we have

$$
F\left(x_{k}\right) \subset F(x)+B(0,1) .
$$

This contradicts (4) and completes the proof of Proposition 3.

In our study, we apply the following two theorems (see Theorem 4 on page 13 of [17] and Theorem 1 on page 60 of [17], respectively).

Theorem 1. Let $I \subset R^{1}$ be an interval, for every $k \in \mathcal{N}, x_{k}: I \rightarrow R^{n}$ be an a. c. function such that, for each real number $t \in I$, the sequence $\left\{x_{k}(t)\right\}_{k=1}^{\infty}$ is bounded and let a positive function $c \in L^{1}(I)$ satisfy

$$
\left\|x_{k}^{\prime}(t)\right\| \leq c(t)
$$

for a. e. real numbers $t \in I$ and every $k \in \mathcal{N}$. Then, there are a subsequence $\left\{x_{k_{i}}\right\}_{i=1}^{\infty}$ and an a.c. function $x: I \rightarrow R^{n}$ such that $x_{k_{i}}$ converges to the function $x$ uniformly over compact subsets of the interval I and that the functions $x_{k_{i}}^{\prime}$ converges weakly to the function $x^{\prime}$ in $L^{1}\left(I ; R^{n}\right)$ as $i \rightarrow \infty$.

Theorem 2. Let $I \subset R^{1}$ be an interval, for every $k \in \mathcal{N}, x_{k}: I \rightarrow X$ and $y_{k}: I \rightarrow R^{n}$ be Lebesgue measurable functions such that, for $a$. e. real numbers $t \in I$ and every open neighborhood $N$ of zero in the space $R^{n} \times R^{n}$, there is $k_{0}(t, N) \in \mathcal{N}$ such that, for every natural number $k \geq k_{0}(t, N)$,

$$
\left(x_{k}(t), y_{k}(t)\right) \in \operatorname{graph}(F)+N .
$$

Let us suppose that $x_{k}$ converges $a$. e. to the function $x: I \rightarrow R^{n}$ and $y_{k} \in L^{1}\left(I ; R^{n}\right)$ converges to $y$ weakly in $L^{1}\left(I ; R^{n}\right)$ as $k \rightarrow \infty$. Then, for a. e. real number $t \in I$,

$$
(x(t), y(t)) \in \operatorname{graph}(F) .
$$

Proposition 3 implies the next proposition.

Proposition 4. Let us assume that $T>0$ and $x:[0, T] \rightarrow X$ is an a. c. function which satisfies

$$
x^{\prime}(t) \in F(x(t)), t \in[0, T] \text { a.e. } .
$$

Then, the function $x$ is Lipschitz on $[0, T]$.

In addition to (A1), the following assumption (A2) is assumed to be satisfied everywhere below.

(A2) For every positive number $M$, there is a positive number $M_{0}$ such that, for every positive number $T$ and each function $x \in \mathcal{A}(0, T)$ which satisfies $\|x(0)\| \leq M$, the equation $\|x(t)\| \leq M_{0}$ is valid for every number $t \in[0, T]$.

Note that (A2) holds for models of economic growth which are prototypes of our dynamical system $[7,9]$.

The next result, which is deduced from Theorems 1 and 2, plays an important role in our study.

Theorem 3. Let us assume that $-\infty<T_{1}<T_{2}<\infty$, for each $k \in \mathcal{N}, x_{k} \in \mathcal{A}\left(T_{1}, T_{2}\right)$ and that the set $\left\{x_{k}\left(T_{1}\right): k=1,2, \ldots\right\}$ is bounded. Then, there exist a subsequence $\left\{x_{i_{k}}\right\}_{k=1}^{\infty}$ and a trajectory $x \in \mathcal{A}\left(T_{1}, T_{2}\right)$ such that $x_{i_{k}}$ converges to $x$ as $k \rightarrow \infty$ uniformly on $\left[T_{1}, T_{2}\right]$ and $x_{i_{k}}^{\prime}$ converges to $x^{\prime}$ as $k \rightarrow \infty$ weakly in $L^{1}\left(\left[T_{1}, T_{2}\right] ; R^{n}\right)$. 
Proof. There exists $M_{0}>0$ such that

$$
\left\|x_{k}\left(T_{1}\right)\right\| \leq M_{0}, k=1,2, \ldots
$$

By (6) and (A2), there is a positive number $M_{1}$ for which

$$
\left\|x_{k}(t)\right\| \leq M_{1} \text { for every number } t \in\left[T_{1}, T_{2}\right] \text { and every } k \in \mathcal{N} .
$$

It follows, from Proposition 3 and Equations (1) and (7), that there is a positive number $M_{2}$ for which

$$
\left\|x_{k}^{\prime}(t)\right\| \leq M_{2} \text { for a. e. } t \in\left[T_{1}, T_{2}\right] \text { and all } k \in \mathcal{N} .
$$

Theorem 1 and Equation (8) imply that there exist a subsequence $\left\{x_{k_{i}}\right\}_{i=1}^{\infty}$ and an a. c. function $x:\left[T_{1}, T_{2}\right] \rightarrow R^{n}$ such that $x_{k_{i}}$ converges to $x$ uniformly over $\left[T_{1}, T_{2}\right]$ as $i \rightarrow \infty$ and $x_{k_{i}}^{\prime}$ converges weakly to $x^{\prime}$ in $L^{1}\left(\left[T_{1}, T_{2}\right] ; R^{n}\right)$ as $i \rightarrow \infty$. Combined with Theorem 2, this convergence implies that

$$
x^{\prime}(t) \in F(x(t)), t \in\left[T_{1}, T_{2}\right] \text { a. e.. }
$$

Theorem 3 is proved.

\section{The Results}

We begin with the next theorem. It will be proved in Section 3.

Theorem 4. Let $C \subset X$ be a nonempty closed bounded set. Then, the following properties are equivalent:

(1) There exists a function $x \in \mathcal{A}(0, \infty)$ such that $x(0) \in C$.

(2) For every $k \in \mathcal{N}$, there exists a function $x_{k} \in \mathcal{A}\left(0, T_{k}\right)$ such that

$$
\lim _{k \rightarrow \infty} T_{k}=\infty
$$

and $x_{k}(0) \in C$ for every $k \in \mathcal{N}$.

Corollary 1. The following properties are equivalent:

(1) There exists a function $x \in \mathcal{A}(0, \infty)$.

(2) For every $k \in \mathcal{N}$, there is a function $x_{k} \in \mathcal{A}\left(0, T_{k}\right)$ such that

$$
\lim _{k \rightarrow \infty} T_{k}=\infty
$$

and

$$
\sup \left\{\left\|x_{k}(0)\right\|: k=1,2, \ldots\right\}<\infty .
$$

Corollary 2. Let $\xi \in X$. Then, the following properties are equivalent:

(1) There exists a function $x \in \mathcal{A}(0, \infty)$ such that $x(0)=\xi$.

(2) For every $k \in \mathcal{N}$, there is a function $x_{k} \in \mathcal{A}\left(0, T_{k}\right)$ such that

$$
\lim _{k \rightarrow \infty} T_{k}=\infty
$$

and $x_{k}(0)=\xi$ for all $k \in \mathcal{N}$.

In the sequel, we assume that there exists a function $x \in \mathcal{A}(0, \infty)$.

We define

$\Omega(F)=\{z \in X:$ for every positive number $\epsilon$ there is a function $x \in \mathcal{A}(0, \infty)$ 


$$
\text { for which } \left.\liminf _{t \rightarrow \infty}\|z-x(t)\| \leq \epsilon\right\} .
$$

In view of (A2), $\Omega(F) \neq \varnothing$. Evidently, $\Omega(F)$ is a closed subset of $X$. In the literature, the set $\Omega(a)$ is called a global attractor of $a$. Note that, in [1,2], $\Omega(a)$ is called a turnpike set of $a$.

For every point $x \in R^{n}$ and every nonempty set $E \subset R^{n}$, we define

$$
\rho(x, E)=\inf \{\|x-y\|: y \in E\} .
$$

The following proposition is proved in Section 3.

Proposition 5. For every function $x \in \mathcal{A}(0, \infty)$,

$$
\lim _{s \rightarrow \infty} \rho(x(s), \Omega(F))=0 .
$$

The following theorem is proved in Section 4.

Theorem 5. Let $\epsilon, M$ be positive real numbers. Then, there is a positive number $T(M, \epsilon)$ such that for every function $x \in \mathcal{A}(0, T)$ which satisfies

$$
\|x(0)\| \leq M, T>T(M, \epsilon)
$$

there is a number $a \in[0, T-M]$ for which

$$
\rho(x(s), \Omega(F)) \leq \epsilon \text { for all } s \in[a, a+M] .
$$

The following proposition is proved in Section 5.

Proposition 6. Let $\xi \in \Omega(F)$. Then, there exists $x \in \mathcal{A}(-\infty, \infty)$ such that $x(0)=\xi$ and

$$
\sup \left\{\|x(t)\|: t \in R^{1}\right\}<\infty .
$$

The following theorem, which is proved in Section 6, is our main result.

Theorem 6. The following properties are equivalent:

(1) If $x \in \mathcal{A}(-\infty, \infty)$ satisfies

$$
\sup \left\{\|x(t)\|: t \in R^{1}\right\}<\infty,
$$

then $x(t) \in \Omega(F)$ for all $t \in R^{1}$.

(2) For each $\epsilon, M>0$, there exists $L>0$ such that, for each $x \in \mathcal{A}(0, T)$ which satisfies $T>2 L$ and $\|x(0)\| \leq M$, the inequality

$$
\rho(x(t), \Omega(F)) \leq \epsilon
$$

holds for all $t \in[L, T-L]$.

Properties (1) and (2) usually hold for models of economic dynamics, which are prototypes of our dynamical system $[1,2,8,9]$. In particular, it holds for the von NeumannGale model generated by a monotone process of convex type which was studied in [18].

Property (2) is the turnpike property, which is well known in mathematical economics. It was discovered by Samuelson in 1948 (see [19]) and further analyzed for optimal trajectories of models of economic dynamics. See, for example, $[2,8,9]$ and the references mentioned there. Recently, it was shown that the turnpike phenomenon holds for many important classes of problems arising in various areas of research [7,9-16]. For related infinite horizon problems, see [9,20-27]. 
The next result is proved in Section 7.

Proposition 7. Let us assume that property (1) of Theorem 6 holds and that $x \in \mathcal{A}(0, \infty)$ satisfies $x(0) \in \Omega(F)$. Then, $x(t) \in \Omega(F)$ for all $t \geq 0$.

The following theorem is our second main result, which is also proved in Section 7. It shows that, if the starting point of the trajectory is closed to the turnpike, then the trajectory leaves the turnpike only when $t$ is closed to the right endpoint $T$ of the domain.

Theorem 7. Let us suppose that property (1) of Theorem 6 holds and that $\epsilon, M$ are positive real numbers. Then, there exist positive real numbers $\delta, L$ such that, for every function $x \in \mathcal{A}(0, T)$ satisfying $T>L$ and

$$
\|x(0)\| \leq M \text { and } \rho(x(0), \Omega(F)) \leq \delta
$$

the inequality

$$
\rho(x(t), \Omega(F)) \leq \epsilon
$$

is valid for every $t \in[0, T-L]$.

Now, we show that, if the set-valued mapping $F$ has a certain symmetric property, then the turnpike $\Omega(F)$ possesses the corresponding symmetric property. Let us assume that $A: R^{n} \rightarrow R^{n}$ is a linear invertible mapping such that $A^{2}$ is the identity mapping in $R^{n}$. Clearly, $A=A^{-1}$. Let us assume that $A(X)=X$ and

$$
A(F(x))=F(A x)), x \in X .
$$

Let $x \in \mathcal{A}(0, \infty)$. Then, for a. e. $t \geq 0$,

$$
(A x(t))^{\prime}=A x^{\prime}(t) \in A(F(x(t))=F(A x(t))
$$

and $A x \in \mathcal{A}(0, \infty)$. This implies that

$$
A(\Omega(F)) \subset \Omega(F) .
$$

Since $A^{2}$ is the identity mapping in $R^{n}$, the inclusion above implies that

$$
\Omega(F)=A^{2}(\Omega(F)) \subset A(\Omega(F))=\Omega(F) .
$$

Thus, $A(\Omega(F))=\Omega(F)$.

\section{Proofs of Theorem 4 and Proposition 5}

Proof of Theorem 4. Clearly, (1) implies (2). Let us assume that (2) holds and that, for every $k \in \mathcal{N}, x_{k} \in \mathcal{A}\left(0, T_{k}\right)$ satisfies

$$
\lim _{k \rightarrow \infty} T_{k}=\infty
$$

and $x_{k}(0) \in C$ for all $k \in \mathcal{N}$. By Theorem 3 and the relations above, extracting subsequences and using the diagonalization process, we obtain that there exist a subsequence $\left\{x_{k_{i}}\right\}_{i=1}^{\infty}$ and $x \in \mathcal{A}(0, \infty)$ such that $x_{k_{i}}$ converges to $x$ as $i \rightarrow \infty$ uniformly on $[0, p]$ for every $p \in \mathcal{N}$. Thus, (1) holds and (2) implies (1). Theorem 4 is proved.

Proof of Proposition 5. Let $x \in \mathcal{A}(0, \infty)$. By (A2),

$$
\sup \{\|x(t)\|: t \in[0, \infty)\}<\infty .
$$

Evidently, we only need to verify that

$$
\lim _{s \rightarrow \infty} \rho(x(s), \Omega(F))=0 .
$$


Let us assume the contrary. Then, there exist $\epsilon>0$ and a sequence of positive numbers $t_{k} \rightarrow \infty$ as $k \rightarrow \infty$ such that

$$
\rho\left(x\left(t_{k}\right), \Omega(F)\right)>\epsilon, k=1,2, \ldots
$$

In view of (10), we may assume that the sequence $\left\{x\left(t_{k}\right)\right\}_{k=1}^{\infty}$ converges. Clearly, its limit belongs to $\Omega(F)$. This contradicts (11) and proves Proposition 5 .

\section{Proof of Theorem 5}

Let us assume that the theorem is not true. Then, for every $k \in \mathcal{N}$, there exist

$$
T_{k}>M+k, x_{k} \in \mathcal{A}\left(0, T_{k}\right)
$$

for which

$$
\left\|x_{k}(0)\right\| \leq M
$$

and, for every $a \in\left[0, T_{k}-M\right]$,

$$
\sup \left\{\rho\left(x_{k}(t), \Omega(F)\right): t \in[a, a+M]\right\}>\epsilon .
$$

In view of (A2) and (3.13), there exists a positive number $M_{0}$ for which

$$
\left\|x_{k}(t)\right\| \leq M_{0}, t \in\left[0, T_{k}\right], k \in \mathcal{N} .
$$

By Theorem 3 and Equations (12) and (15), extracting subsequences and using the diagonalization process, we obtain that there exist a subsequence $\left\{x_{k_{j}}\right\}_{i=1}^{\infty}$ and $x \in \mathcal{A}(0, \infty)$ such that, for every $p \in \mathcal{N}$,

$$
x_{k_{j}} \text { converges to } x \text { as } j \rightarrow \infty \text { uniformly on }[0, p] .
$$

By Proposition 5,

$$
\lim _{s \rightarrow \infty} \rho(x(s), \Omega(F))=0 .
$$

Therefore, there is a positive number $T_{0}$ such that, for every real number $t \geq T_{0}$,

$$
\rho(x(t), \Omega(F)) \leq \epsilon / 4 .
$$

By (16), there is a natural number $k>T_{0}+M$ for which

$$
\left\|x_{k}(t)-x(t)\right\| \leq \epsilon / 2, t \in\left[0, T_{0}+M\right] .
$$

Combined with (17), the equation above implies that

$$
\rho\left(x_{k}(t), \Omega(F)\right) \leq 3 \epsilon / 4, t \in\left[T_{0}, T_{0}+M\right] .
$$

This contradicts (14) and proves Theorem 5.

\section{Proof of Proposition 6}

By definition (9), for each $k \in \mathcal{N}$, there is a function $x_{k} \in \mathcal{A}(0, \infty)$ for which

$$
\liminf _{t \rightarrow \infty}\left\|x_{k}(t)-\xi\right\| \leq 1 / k
$$

By (18), we may assume, without loss of generality, that

$$
\left\|x_{k}(0)\right\| \leq\|\xi\|+1, k=1,2, \ldots
$$


Assumption (A2) and (19) imply that there is a positive number $M_{1}$ for which

$$
\left\|x_{k}(t)\right\| \leq M_{1}, t \in[[0, \infty), k=1,2, \ldots
$$

Let $k \in \mathcal{N}$. By (18), there is a real number $t_{k}$ for which

$$
t_{k}>k,\left\|x_{k}\left(t_{k}\right)-\xi\right\| \leq 1 / k .
$$

We define

$$
y_{k}(t)=x_{k}\left(t+t_{k}\right), t \in\left[-t_{k}, \infty\right)
$$

Clearly,

$$
y_{k} \in \mathcal{A}\left(-t_{k}, \infty\right)
$$

Equations (20) and (22) imply that

$$
\left\|y_{k}(t)\right\| \leq M_{1}, t \in\left[-t_{k}, \infty\right) .
$$

By (21)-(24), extracting subsequences and using the diagonalization process, we obtain that there exist a subsequence $\left\{y_{k_{j}}\right\}_{i=1}^{\infty}$ and $x \in \mathcal{A}(-\infty, \infty)$ such that, for every $p \in \mathcal{N}$,

$$
y_{k_{j}} \text { converges to } x \text { as } j \rightarrow \infty \text { uniformly on }[-p, p] \text {. }
$$

Equations (21), (22) and (25) imply that

$$
x(0)=\lim _{j \rightarrow \infty} y_{k_{j}}(0)=\lim _{j \rightarrow \infty} x_{k_{j}}\left(t_{k_{j}}\right)=\xi .
$$

By (24) and (25), $\|x(t)\| \leq M_{1}$ for all $t \in R^{1}$. Proposition 6 is proved.

\section{Proof of Theorem 6}

Clearly, (2) implies (1). Let us assume that property (1) holds. We verify that property (2) is also valid.

Let us assume the contrary. Then, there are positive real numbers $\epsilon, M$ and, for each $k \in \mathcal{N}$, there exist $x_{k} \in \mathcal{A}\left(0, T_{k}\right)$ such that

$$
\begin{gathered}
T_{k}>2 k, \\
\left\|x_{k}(0)\right\| \leq M, \\
\sup \left\{\rho\left(x_{k}(t), \Omega(F)\right): t \in\left[k, T_{k}-k\right]\right\}>\epsilon .
\end{gathered}
$$

Assumption (A2) and (27) imply that there is a positive number $M_{1}$ for which

$$
\left\|x_{k}(t)\right\| \leq M_{1}, t \in\left[0, T_{k}\right], k=1,2, \ldots .
$$

Let $k \in \mathcal{N}$. By (28), there is a number

$$
\tau_{k} \in\left[k, T_{k}-k\right]
$$

such that

$$
\rho\left(x_{k}\left(\tau_{k}\right), \Omega(F)\right)>\epsilon \text {. }
$$

We define

$$
y_{k}(t)=x_{k}\left(t+\tau_{k}\right), t \in\left[-\tau_{k}, \infty\right) .
$$

It follows, from (26), (29), (30) and (32), that

$$
y_{k} \in \mathcal{A}\left(-\tau_{k}, \infty\right),
$$




$$
\left\|y_{k}(t)\right\| \leq M_{1}, t \in\left[-\tau_{k}, \infty\right) .
$$

By (33) and Theorem 3, extracting subsequences and using the diagonalization process, we obtain that there exist a subsequence $\left\{y_{k_{j}}\right\}_{i=1}^{\infty}$ and $x \in \mathcal{A}(-\infty, \infty)$ such that, for every $p \in \mathcal{N}$,

$$
y_{k_{j}} \text { converges to } x \text { as } j \rightarrow \infty \text { uniformly on }[-p, p] \text {. }
$$

Property (1) implies that

$$
x(t) \in \Omega(F), t \in R^{1} .
$$

Equations (32) and (34) imply that

$$
x(0)=\lim _{j \rightarrow \infty} y_{k_{j}}(0)=\lim _{j \rightarrow \infty} x_{k_{j}}\left(\tau_{k_{j}}\right) .
$$

Combined with (31), the equation above implies that

$$
\rho(x(0), \Omega(F)) \geq \epsilon .
$$

This contradicts (35). The contradiction we reached proves that property (2) holds. Thus, (1) implies (2). Theorem 6 is proved.

\section{Proofs of Proposition 7 and Theorem 7}

Proof of Proposition 7. Proposition 6 implies that there exists $y \in \mathcal{A}(-\infty, \infty)$ such that

$$
\begin{gathered}
y(0)=x(0), \\
\sup \left\{\|y(t)\|: t \in R^{1}\right\}<\infty .
\end{gathered}
$$

We define

$$
x(t)=y(t), t \in(-\infty, 0] .
$$

Clearly, $x \in \mathcal{A}(-\infty, \infty)$. By (A2) and the equation above,

$$
\sup \left\{\|x(t)\|: t \in R^{1}\right\}<\infty .
$$

It follows, from property (1) of Theorem 6, that $x(t) \in \Omega(F)$ for every $t \in R^{1}$. Proposition 7 is proved.

Proof of Theorem 7. In view of Theorem 6 , there is $L_{0}>1$ such that the following property holds:

(i) for every function $x \in \mathcal{A}(0, T)$ satisfying $T>2 L_{0}$ and $\|x(0)\| \leq M$, the equation

$$
\rho(x(t), \Omega(F)) \leq \epsilon
$$

is valid for every $t \in\left[L_{0}, T-L_{0}\right]$.

Let us assume that our theorem does not hold. Then, for each $k \in \mathcal{N}$, there is $x_{k} \in \mathcal{A}\left(0, T_{k}\right)$ for which

$$
\begin{gathered}
T_{k}>k+2 L_{0},\left\|x_{k}(0)\right\| \leq M, \\
\rho\left(x_{k}(0), \Omega(F)\right) \leq 1 / k
\end{gathered}
$$

and

$$
\sup \left\{\rho\left(x_{k}(t), \Omega(F)\right): t \in\left[0, T_{k}-k-2 L_{0}\right]\right\}>\epsilon .
$$

Assumption (A2) and (36) imply that there is a positive number $M_{1}$ for which

$$
\left\|x_{k}(t)\right\| \leq M_{1}, t \in\left[0, T_{k}\right], k=1,2, \ldots
$$


Let $k \in \mathcal{N}$. In view of (38), there is

$$
\tau_{k} \in\left[0, T_{k}-k-2 L_{0}\right]
$$

such that

$$
\rho\left(x_{k}\left(\tau_{k}\right), \Omega(F)\right)>\epsilon .
$$

Property (i) and Equations (36), (40) and (41) imply that

$$
\tau_{k} \leq L_{0}
$$

By (36), (39) and Theorem 3, extracting subsequences and using the diagonalization process, we obtain that there exist a subsequence $\left\{x_{k_{j}}\right\}_{i=1}^{\infty}$ and $x \in \mathcal{A}(0, \infty)$ such that for every $p \in \mathcal{N}$,

$$
x_{k_{j}} \text { converges to } x \text { as } j \rightarrow \infty \text { uniformly on }[0, p] \text {. }
$$

In view of (37) and (43),

$$
x(0) \in \Omega(F) .
$$

Combined with Proposition 15, the inclusion above implies that

$$
x(t) \in \Omega(F), t \in[0, \infty) .
$$

By (43), there exists $j \in \mathcal{N}$ such that

$$
\left\|x(t)-x_{k_{j}}(t)\right\| \leq \epsilon / 2, t \in\left[0,2 L_{0}\right]
$$

and, in view of (42) and (44),

$$
\rho\left(x_{k_{j}}\left(\tau_{k_{j}}\right), \Omega(F)\right) \leq\left\|x_{k_{j}}\left(\tau_{k_{j}}\right)-x\left(\tau_{k_{j}}\right)\right\| \leq \epsilon / 2
$$

This contradicts (41) and completes the proof of Theorem 7.

Funding: This research received no external funding.

Institutional Review Board Statement: Not applicable.

Informed Consent Statement: Not applicable.

Data Availability Statement: Not applicable.

Conflicts of Interest: The author declares no conflict of interest.

\section{References}

1. Rubinov, A.M. Turnpike sets in discrete disperse dynamical systems. Sib. Math. J. 1980, 21, 136-146.

2. Rubinov, A.M. Multivalued Mappings and Their Applications in Economic Mathematical Problems; Nauka: Leningrad, Russia, 1980.

3. Zaslavski, A.J. Turnpike sets of continuous transformations in compact metric spaces. Sib. Math. J. 1982, 23, 136-146. [CrossRef]

4. Zaslavski, A.J. Uniform convergence to global attractors for discrete disperse dynamical systems. Nonlinear Dyn. Syst. Theory 2007, 4, 315-325.

5. Zaslavski, A.J. Convergence of trajectories of discrete dispersive dynamical systems. Commun. Math. Anal. 2008, 4, 10-19.

6. Zaslavski, A.J. Structure of trajectories of discrete dispersive dynamical systems. Commun. Math. Anal. 2009, 6, 1-9.

7. Zaslavski, A.J. Optimal Control Problems Related to the Robinson-Solow-Srinivasan Model; Monographs in Mathematical Economics; Springer: Berlin/Heidelberg, Germany, 2020.

8. Makarov, V.L.; Rubinov, A.M. Mathematical Theory of Economic Dynamics and Equilibria; Springer: New York, NY, USA, 1977.

9. Zaslavski, A.J. Turnpike Properties in the Calculus of Variations and Optimal Control; Optimization and Its Applications; Springer: New York, NY, USA, 2006.

10. Damm, T.; Grune, L.; Stieler, M.; Worthmann, K. An exponential turnpike theorem for dissipative discrete time optimal control problems. Siam J. Control. Optim. 2014, 52, 1935-1957. [CrossRef]

11. Gugat, M. A turnpike result for convex hyperbolic optimal boundary control problems. Pure Appl. Funct. Anal. 2019, 4, 849-866. 
12. Gugat, M.; Trelat, E.; Zuazua, E. Optimal Neumann control for the 1D wave equation: Finite horizon, infinite horizon, boundary tracking terms and the turnpike property. Syst. Control. Lett. 2016, 90, 61-70. [CrossRef]

13. Mammadov, M. Turnpike theorem for an infinite horizon optimal control problem with time delay. Siam J. Control. Optim. 2014, 52, 420-438. [CrossRef]

14. Porretta, A.; Zuazua, E. Long time versus steady state optimal control. SIAM J. Control Optim. 2013, 51, 4242-4273. [CrossRef]

15. Trelat, E.; Zhang, C.; Zuazua, E. Optimal shape design for 2D heat equations in large time. Pure Appl. Funct. Anal. 2018, 3, 255-269.

16. Trelat, E.; Zuazua, E. The turnpike property in finite-dimensional nonlinear optimal control. J. Differ. Equations 2015, 218, 81-114. [CrossRef]

17. Aubin, J.-P.; Cellina, A. Differential Inclusions. Set-Valued Maps and Viability Theory; Springer: Berlin/Heidelberg, Germany, 1984.

18. Rockafellar, R.T. Monotone Processes of Convex and Concave Type; Memoirs of the American Mathematical Society: Providence, RI, USA, 1967; Volume 77.

19. Samuelson, P.A. A catenary turnpike theorem involving consumption and the golden rule. Amer. Econom. Rev. 1965, 55, 486-496.

20. Aseev, S.M.; Krastanov, M.I.; Veliov, V.M. Optimality conditions for discrete-time optimal control on infinite horizon. Pure Appl. Funct. Anal. 2017, 2, 395-409.

21. Bachir, M.; Blot, J. Infinite dimensional infinite-horizon Pontryagin principles for discrete-time problems. Set-Valued Var. Anal. 2015, 23, 43-54. [CrossRef]

22. Bachir, M.; Blot, J. Infinite dimensional multipliers and Pontryagin principles for discrete-time problems. Pure Appl. Funct. Anal. 2017, 2, 411-426.

23. Blot, J.; Hayek, N. Infinite-Horizon Optimal Control in the Discrete-Time Framework; Briefs in Optimization; Springer: New York, NY, USA, 2014.

24. Carlson, D.A.; Haurie, A.; Leizarowitz, A. Infinite Horizon Optimal Control; Springer: Berlin/Heidelberg, Germany, 1991.

25. Gaitsgory, V.; Grune, L.; Thatcher, N. Stabilization with discounted optimal control. Syst. Control. Lett. 2015, 82, 91-98. [CrossRef]

26. Gaitsgory, V.; Mammadov, M.; Manic, L. On stability under perturbations of long-run average optimal control problems. Pure Appl. Funct. Anal. 2017, 2, 461-476.

27. Sagara, N. Recursive variational problems in nonreflexive Banach spaces with an infinite horizon: An existence result. Discrete Contin. Dyn. Syst. Ser. S 2018, 11, 1219-1232. [CrossRef] 